\title{
La epistemología de la Educación Física en relación al currículum de Secundaria en la Ley orgánica de Educación The epistemology of Physical Education in relation to the curriculum in Secondary Organic Law of Education
}

\author{
Antonio Baena Extremera*, Antonio Granero Gallegos**, Manuel Gómez López* \\ *Universidad de Murcia (España); **Universidad de Almería (España) \\ Resumen: Toda disciplina debe definir qué es aquello que la ocupa y preocupa, para, utilizando el saber conocido (la epistemología), ser capaz de avanzar \\ y generar un conocimiento específico que sea coherente y complementario con el generado por otras disciplinas científicas. El objetivo de este artículo \\ es hacer una aproximación epistemológica al currículum de Educación Física en la etapa de Secundaria, desde sus orígenes hasta la nueva Ley Educativa. \\ Palabras clave: Epistemología, Educación Física, Currículum, Educación Secundaria.
}

Abstract: All discipline must define what is and what the deals are concerned, for using knowledge known, (the epistemology), being able to move forward and create a specific knowledge that is consistent with and complementary to that generated by other scientific disciplines. The aim of this article is to make an epistemological approach to currículum of Physical Education at the Secondary School, from its origins to the new Education Law. Key words: Epistemology, Physical Education, Curriculum, Secondary School.

\section{Introducción a la Epistemología de la Educación Física.}

El término Epistemología, proviene del griego «episteme» (conocimientos o saber científico) y del término «logos» (tratado). El término epistemología fue introducido por F. Ferrier (1808-1864), es sinónimo de «gnoseología», vocablo éste caído en desuso por sus connotaciones escolástico-especulativos.

El D.R.A.E. define Epistemología, como la doctrina de los fundamentos y métodos del conocimientos científico. Se ocupa por tanto, de dar respuesta a toda la problemática relacionada con la investigación científica, cómo los problemas lógicos, semánticos, metodológicos, axiológicos, ontológicos, etc.

Bachelard (1978, citado en Rodríguez, 2003) la define como la aplicabilidad racional de pensamientos, reflexiones y métodos que operan para vencer el límite del saber conocido. Diferencia entre ÁREAS EPISTEMOLÓGICAS definidas como parcelas de saber científico donde se ubican cada una de las diferentes disciplinas en función del objeto de estudio, y ACTOS EPISTEMOLÓGICOS definidos como el acto de investigar, que necesita de las siguientes fases:

-Romper con la realidad inmediata

-Construcción de un objeto científico

-Contrastación del objeto con los hechos

Lagardera (1989) define la epistemología como los discursos, ensayos y doctrina, que tienen como base los métodos y fundamentos del conocimiento científico, así como determinados métodos y parcelas o regiones epistemológicas que son atribuidas a una determinada ciencia.

Actualmente, la epistemología:

-Se ocupa fundamentalmente de cuestiones relativas a la teoría de la ciencia y la evolución y configuración de los distintos saberes científicos relativos a una determinada parcela del conocimiento.

-Es una rama de la filosofía que estudia la investigación científica y su producto.

-Se ocupa de los problemas filosóficos que se presentan en el curso de la investigación: problemas lógicos, semánticos,...

-Reflexiona acerca de las teorías formuladas, método de investigación utilizado, de las hipótesis, así como de las leyes que de ellas se pudieran desprender (Bunge, 1985).

Fecha recepción: 11-10-08 - Fecha envío revisores: 12-10-08- Fecha de aceptación: 23-12-08 Correspondencia: Antonio Baena Extremera

C/ Granada, 6

8217 Güevéjar (Granada)

E-mail: abaenaextrem $@$ yahoo.es
-Propone soluciones a dichos problemas consistentes en teorías rigurosas, inteligibles y adecuadas a la realidad de la investigación.

La epistemología de la Educación Física (EF), debe proporcionar una sistemática que permita la solución de los diferentes problemas surgidos en relación con la Educación Física y la actividad física y dar respuesta a las siguientes cuestiones:

-¿Es la Educación Física una ciencia? No es una ciencia única, aunque si tiene un único objeto de estudio, dicho objeto es el hombre en movimiento. Si bien, el término movimiento es muy amplio e indeterminado, que permite delimitar nuestro campo de acción, lo que nos remite a la praxis física. Las actividades físicas o acciones con significación práxica a las que hacemos referencia son las siguientes:

\section{1. * *as físico-deportivas \\ *Las físico-lúdico-recreativas \\ * Las físico-expresivas \\ *Las físico-aprehensiva (objeto de la Educación Física enten- dida como disciplina pedagógica).}

-¿Quétipo de ciencia es y cual es su naturaleza científica interna? La EF está compuesta por muchas ciencias, al estudiar la praxis desde diferentes ámbitos científicos. La Epistemología de la EF debe proporcionar una Teoría General de la EF con capacidad para vertebrar, estructurar y sistematizar todos los conocimientos relacionados con la misma.

Por tanto, la Epistemología de la EF se encarga de estudiar los conocimientos que existen en torno a la EF y la relación de estos con la práctica.

Para Pedraz (1988), la Epistemología de la EF tendría dos tareas:

-Determinar las ciencias y disciplinas que configuran el campo pedagógico

-Formular una teoría general o un contorno filosófico que permita: *Definir el concepto de EF.

* Organizar todas las aproximaciones que se produzca en este campo del saber.

*Alcanzar un consenso terminológico que facilite la investigación y el estudio.

En el transcurso de la historia de la epistemología del siglo XX se han contemplado una diversidad de enfoques con respecto al método científico, entre los que se destacan los siguientes (Garay y Hernández, 2005):

1.El neopositivismo del Círculo de Viena.

2. El falsacionismo de Karl Popper.

3. El concepto de paradigma de Thomas Kuhn.

4. Los programas de investigación cientifica de Imre Lakatos.

5. Las tradiciones de investigación de Larry Laudan.

6. El anarquismo metodológico de Karl Feyerabend.

7. El constructivismo. 
El Diseño Curricular Base señala que la epistemología de la EF es algo que esta en desarrollo debido a la discusión en torno a cuales son los objetivos de la EF y qué disciplinas la integran. Prueba de ello es el Real Decreto 1631/2006 de 29 de Diciembre, establece las enseñanzas mínimas de Educación Secundaria, que han modificado los objetivos, contenidos y criterios de evaluación establecidos por las leyes orgánicas anteriores. En la elaboración de este Real Decreto, se estima entre otros aspectos, la importancia de la reflexión acerca de la influencia de las fuentes del currículum, donde encontramos la Fuente Epistemológica. El análisis de las disciplinas o materias debe permitir diferenciar aquellos conocimientos que son importantes de los que son secundarios, también debe permitir el conocimiento de su estructura interna y de las diferentes relaciones existentes entre ellos. Se debe tener en cuenta la evolución científica de los diferentes contenidos, su lógica interna y su estructura para poder llegar a diseñar actividades de enseñanza-aprendizaje que sean verdaderamente significativas y que, a la vez, respeten la teoría elaborativa. De esta forma será posible llegar a la máxima asimilación de los mismos por los alumnos (Díaz Lucea, 1994:32).

2. El método científico como fuente de conocimiento epistemológico.

El método científico lo podemos definir como: «El procedimiento para tratar un conjunto de problemas, ajustándose al método general de la ciencia. El método científico es un proceso sistemático y organizado por medio del cual se adquieren conocimientos objetivos del mundo y su producto es el conocimiento científico. La consideración de ciencia no depende del objeto de estudio, ni de las características propias de la disciplina, sino de lautilización correcta del método científico» (Gutiérrez y Oña, 2003).

Como Bunge (1985) señala muy acertadamente, se podría entender como la manera de proceder característica de la ciencia se ha dado en llamar método científico. El nombre es ambiguo. Por una parte, es merecido porque tal método existe y es eficaz.

La construcción de un objeto científico supone una reflexión de primer orden, en cuanto que necesitamos definir qué es aquello que queremos o necesitamos saber. Cuál será el objeto de nuestro estudio, preocupaciones o pesquisas científicas. La investigación científica se organiza de hecho en torno de objetos construidos que no tienen nada en común con aquellas unidades delimitadas por la percepción ingenua (Bourdieu y col., 1976).

En relación a laEducación Física como ciencia, deberíamos diferenciar el objetivo de conocimiento de nuestra área:

- Desde la visión de la Educación Física como área de saber: Diferentes autores (Schmidt, Simonet, Parlebás, citados por Gimeno, 1998, p.11) coinciden en designar con el término acción motriz o conducta motriz el objeto de estudio de la Educación Física. El concepto de acción motriz, en palabras de Gimeno, permite la confluencia de una gama de factores observables, psicológicos y fisiológicos, que se alejan del concepto de movimiento, entendido como una mera habilidad mecánica; la acción motriz es, pues, una ejecución intencional, guiada racionalmente e interactiva con el resto de dimensiones de la personalidadhumana.

- Desde la visión de la Educación Física como área curricular: La presente concepción curricular entiende que la EF es un área dispuesta y organizada para colaborar en la educación integral de los niños y niñas, que utiliza el cuerpo y el movimiento como ejes de la acción educativa y que, por tanto, presenta como objeto u objetivo de su proceder la formación total del ser humano. Ej. Diseño Curricular Base deAndalucía: «En consecuencia hay que situar a la Educación Física en el contexto general del desarrollo de la persona, dándole el verdadero sentido de educar a través de lo físico o, lo que es lo mismo, sirviéndose del cuerpo y de sus posibilidades de movimiento para lograr objetivos educativos de carácter más amplio».

A la hora de hablar de las Fuentes de conocimiento en la Educación Física, se debe especificar:
- La Perspectiva multidisciplinar: Las primeras teorizaciones existentes sobre la Educación Física, a finales del siglo XVIII, se elaboran bajo el sustento de la ciencia médica. Anatomía y Fisiología se convierten en sustrato sobre el que se fundan los principales argumentos de la nueva disciplina emergente. Vicente Pedraz, coincide con Arnold (1991) cuando afirma que la educación física ha ampliado el campo de investigación de las ciencias de la educación, desde una triple visión:

a.Diversificando su objeto de estudio a través de la psicología del desarrollo motor o la sociología del deporte.

b.Considerando otras (biología, fisiología y anatomía) que anteriormente se ocuparon de la educación de forma marginal y que ahora participan con más empeño en el campo educativo.

c.Destacando la influencia de otras ciencias no significativas en el ámbito educativo, como la Física a través de biomecánica y la kinesiología.

En definitiva, el objeto y contenido de la EF fue, es y será intervenido por los conocimientos propios de otras ciencias que poco a poco querrán especificar con más precisión la verdadera naturaleza de su aportación.

- El espacio disciplinar de la Educación Física: Para González, citado por Saénz-López (1997, p.22), la EF se constituye como fenómeno integrante de las Ciencias de la Educación. Pero la EF ha de adquirir como finalidad la educación del individuo, en un ámbito de relaciones tanto endógenas como exógenas, sirviéndose de todo componente motriz que posea contenido educativo, y convirtiéndose en un campo del saber autónomo e incardinado en el conjunto de las Ciencias de la Educación.

Es el método el elemento central del conocimiento científico, «método» no ausente en el campo de conocimientos de la EF, y que, según Cagigal (citado por Buñuel, 1997, p.20) comienza a estar dotado de su propia metodología al servicio de un «corpus» en continuo crecimiento. Según González es una actividad cientifica aplicada, una ciencia educativa especializada en el comportamiento motor, en las conductas motrices.

Otros autores como Ommo Grupe no ven tan clara la independencia de la Educación Física como ciencia. Grupe prefiere incardinarla en elámbito de la Ciencias de la Educación, manifestando al respecto: «Las ciencias de la educación parecen estar en situación de abarcar en gran medida, a partir de lo anchuroso de sus planteamientos, los ejercicios fisicos, el deporte, el juego(...) si se concibe asíla educación, entrarán en ella no sólo la educación fisica con su intención educativa... sino también, el juego libre del niño y del hombre maduro...».

Mario Bunge (1985) definía la teoría científica como un conjunto de ideas organizadas lógicamente, comprobables desde la experiencia y por lo tanto verificables en sus premisas y afirmaciones. Así, a diferencia del conocimiento ordinario, no especializado y basado en inducciones, destaca el conocimiento científico que se construye a partir de áreas de conocimiento específicas, soluciona los problemas mediante un método y está ideado para contrastar las soluciones.

Hoy, en un análisis de la situación actual, todas las instituciones tienen como gran obsesión la de producir ciencia. En este discurso, Rodríguez (citado por Buñuel, 1997, p.20), defiende el carácter científico de la EF y lo demuestra cuando cita al numeroso grupo de investigadores y docentes, que presentes en los departamentos de la universidad, están exclusivamente preocupados por seguir avanzando científicamente en este campo del saber.

La polémica se centra en determinar si la EF es ciencia autónoma o dependiente, y posteriormente, determinar cuál es el objeto de estudio de la ciencia de laEF. Cagigal (1968) define el carácter científico de laEF, adscrita a las ciencias pedagógicas: «la EF va estructurando sus conocimientos, se esfuerza por estandarizar internacionalmente los métodos de observación, adquiriendo una sistemática cada vez más específica, $y$ delimita su objeto propio, concretándolo en el hombre en movimiento o en cuanto capaz de movimiento, con todas sus consecuencias culturales».

Parlebás (1981) siguiere un nuevo paradigma: la ciencia de la Praxiologia Motriz, cuyo objeto de conocimiento es la acción motriz. La praxiología motriz tiene por objeto formal el estudio científico de las 
acciones motrices. Este concepto es mucho más amplio que el de conducta motriz, abarcando los procesos de adaptación de las conductas motrices a una situación motriz determinada.

Estudiando a diferentes autores, se entienden cuatro tendencias:

1. La EF no tiene un estatus científico, no es una ciencia sino un elemento más que aparece en la cultura y en la escuela.

2. Se considera que la EF está en un estado precientífico, llegando a ser ciencia en un futuro próximo, cuando cumpliera los siguientes requisitos.

*Tener un cuerpo científico amplio, sistemático y coherente.

*Tener un campo de estudio propio (objeto formal propio) y específico; siendo distinto de las otras ciencias.

3. Se considera que la $\mathrm{EF}$ tiene un carácter científico pero encuadrada dentro de otras disciplinas: pedagogía, medicina, fisiología, etc.

4. Se considera que la EF es una ciencia independiente. Su característica es la «especificidad».

CLASIFICACIÓN DE CIENCIAS QUE APOYAN A LA EF EN FUNCIÓN

\begin{tabular}{|l|l|l|}
\multicolumn{3}{c|}{ DE SU CONTENIDO CIENTÍFICO } \\
\hline \multicolumn{1}{|c|}{ CIENCIAS DE } & \multicolumn{1}{|c|}{ CIENCIAS DE LA } & \multicolumn{1}{c|}{ CIENCIAS MÉDICO- } \\
LIOLUCACIÓN & \multicolumn{1}{c|}{ MOTRICIDAD } & \multicolumn{1}{c|}{ BIOLÓGICAS } \\
- Pedagogía de la EF y el Deporte & - Deportes & - Anatomía funcional \\
- Didáctica de la EF y el Deporte & - Biomecánica deportiva & - Fisiología deportiva \\
- Filosofía de la EF y el Deporte & - Entrenamiento deportivo & - Traumatología \\
- Psicología del Deporte & - Gimnasia & - Medicina preventiva \\
- Sociología del Deporte & - Aprendizaje motor & - Desarrollo motor \\
- Organización de la EF y el Deporte & - Psicomotricidad & $-\ldots$ \\
- Historia de la EF y el Deporte & - Educación física de base & \\
- ... & $-\ldots$ & \\
\hline
\end{tabular}

3. Los Contenidos como componente epistemológico del currículum de Secundaria.

Cesar Coll (1992, p13) define los contenidos como el conjunto de saberes y formas culturales cuya asimilación y apropiación por los alumnos y alumnas se considera esencial para su desarrollo y socialización. Sobre este concepto, Díaz Lucea (1994) afirma que son el conjunto de formas culturales y de saberes seleccionados para formar parte del área en función de los objetivos generales de área».

Los contenidos (Qué enseñar) constituyen el marco de referencia o sustrato conformado para el desarrollo de los objetivos propuestos en el plan educativo. La división formal en tres ámbitos: conceptos, procedimientos y actitudes intenta justificar que el desarrollo motriz debe tratarse en comunión con el desarrollo cognitivo, afectivo-social y moral.

Dentro del currículum, los contenidos se agrupan el Bloques de contenidos, los cuales son entendidos como agrupaciones de contenidos en los que se presenta al profesor la información relativa a lo que ha de trabajar durante toda una etapa educativa. Ofrecen aquellos contenidos más adecuados para desarrollar las capacidades indicadas en los objetivos generales de área. Están dictados por la Administración (D.C.B.).

- El contenido es una decisión preactiva a tomar: ¿Qué enseñar?

- El contenido es el medio para conseguir el objetivo.

Aunque aparezcan presentados en núcleos o bloques de contenidos, el equipo docente tiene el compromiso de idear nuevas formas de organización en consonancia con la propuesta integral (intradisciplinariedad, interdisciplinariedad y transversalidad) del modelo educativo. En la Reforma aparecen 3 grandes categorías de contenidos:

A) Contenidos referidos a conceptos: se refieren a hechos, conceptos y principios o sistemas conceptuales:

* Hechos: recogen acontecimientos o situaciones relevantes. Ejem plo: La Olimpiada del año 1992 se celebró en Barcelona.

*Conceptos: designan un conjunto de hechos o símbolos que poseen características comunes. Ejemplo: el pase, el desmarque. Pero no como acción de ejecutarlos, sino como conceptos, definición.

*Principios o sistemas conceptuales: reflejan la relación existente entre los conceptos, como los cambios que se producen en un objeto o situación se relacionan con los cambios que se producen en otro objeto o situación. Ejemplo: los sistemas de defensa en baloncesto. La adaptación al ejercicio.
B) Contenidos referidos a procedimientos: son los aspectos culturales (en Educación Física), que imponen un conjunto de acciones ordenadas y orientadas a la consecución de una meta, y que son realizados por los alumnos en su aprendizaje:

* Acciones complejas y simples, fisicas y mentales.

* Habilidades.

* Técnicas.

* Estrategias que llevan a un fin.

C) Contenidos referidos a las actitudes: según Sarabia (1992) son tendencias adquiridas y duraderas para evaluar de un modo determinado un objeto, suceso o situación y para actuar en consonancia con dicha evolución. Son los aspectos culturales referidos a:

* Valores: principio formativo que regula el comportamiento de las personas en cualquier momento o situación. Ejemplo: las conservación de la naturaleza. La deportividad.

* Normas: son la concreción de los valores, reglas de conductas que han de respetar las personas en determinadas situaciones. Las normas obligan a que la actitud sea siempre positiva. Para conseguir el valor las conductas llevan siempre implicadas al formularlas verbos. Ejemplo: «siempre» hay que limpiar la zona de zarzales y matorrales.

* Actitudes: reflejan el respeto por las normas y valores. Poseen dos acepciones:

Actitud como comportamiento (Osgood, 1949):

-Además se expresan conductas hacia algo o alguien.

Más valiosa y objetiva.

Actitud como opinión (Krech y cols., 1978):

-Motivación que se supone orienta a la acción.

Conocer y valorar los hechos.

Actualmente en España la concepción epistemológica del área de Educación Física escolar es la que nos aporta la LOE, ya que la LOGSE y LOCE quedaron derogadas, dejando en vigor únicamente la Orden 10 de Agosto del 2007 por la que se desarrolla el currículo correspondiente a la Educación Secundaria Obligatoria en Andalucía y el Decreto 231/ 2007 por el que se establece la ordenación y las enseñanzas correspondientes a la misma etapa. En esta legislación, el área de Educación Física, goza de un puesto de igualdad como obligatoria en los planes de estudio de primaria y secundaria, con un curriculum oficial expresado en objetivos, contenidos, criterios de evaluación y principios metodológicos generales.

La selección de contenidos así como su organización en diferentes núcleos temáticos y su secuencia en los distintos ciclos o niveles de la etapa, debe entenderse como un referente inicial a partir del cual los centros y el profesorado deberán elaborar sus propios proyectos curriculares y programaciones de aula atendiendo a la singularidad de su alumnado. Se pueden considerar así diversos grados de profundización, abordándolos en distintos momentos, integrándolos con otros contenidos, presentándolos en unidades didácticas interdisciplinares o adaptándolos al alumnado con necesidades educativas especiales.

Los contenidos han de tomar como referencia, al igual que los demás elementos del currículo, la caracterización de aquellos que pueden ser comunes para todas las áreas, al objeto de contribuir a la consecución de los fines y metas que se pretenden alcanzar al concluir esta etapa, sin que se resienta el carácter integrador y pluridisciplinar de la Educación Secundaria Obligatoria.

En el RD 1631/06 de 29 de Diciembre por el que se establecen las enseñanzas mínimas correspondientes a la ESO, se presentan los siguientes bloques de contenidos:

\section{1.- CONDICION FÍSICA Y SALUD}

Existe, pues, para cada individuo una condición física natural que responde a sus características propias y a su evolución, que debe ser cuidada, mejorada y potenciada para favorecer un incremento de la salud y de la calidad de vida. Es lo que se conoce como condición físicasalud. La mejora global y especializada de cualidades finas y habilidades específicas, requiere un segundo nivel en función de las necesidades y características de cada sujeto, haciendo referencia a niveles cualitativos y cuantitativos. A esta perspectiva se le suele denominar condición física-rendimiento. El cuidado corporal, entendido en su más amplio sentido, debe constituir un contenido que se encuentre presente de 
forma constante en la práctica de la educación física. Es necesario potenciar el trabajo en torno a las rutinas propias de la higiene, y otras relacionadas de forma más concreta con la ejercitación física, cumplimiento de normas y adopción de medidas de seguridad. Se pretende que el alumnado conozca la relación existente entre educación física y salud, así como la influencia de ambas en un correcto mantenimiento corporal.

\section{2.- JUEGOS Y DEPORTES}

La actividad lúdica y deportiva se ajusta a los intereses del alumnado y evoluciona en función de los mismos, constituyendo una motivación que favorece el acercamiento natural a la práctica normalizada de la actividad física. La atención a las diferentes edades requiere considerar una concepción del juego y del deporte que propicie el diseño, la planificación y la aceptación voluntaria de reglas facilitando la organización colectiva de situaciones en las que participe el alumnado. La progresión en la complejidad de las normas determina diferentes formas lúdicas y deportivas asociadas al propio psiquismo y a diferentes niveles de adaptación social.

Debe entenderse la práctica lúdica vinculada a la cultura circundante que, en el caso de la comunidad andaluza, aporta multitud de tradiciones y manifestaciones propias de indudable riqueza. Por tanto, hay que tener en cuenta la importancia de que el alumnado conozca y practique juegos tradicionales, como vínculo y disfrute del patrimonio cultural de nuestra Comunidad.

\section{3.-EXPRESIONCORPORAL}

El movimiento y sus cualidades plásticas, asociados a los dominios de posibilidades perceptivas y de coordinación, determinan en gran medida las capacidades expresivas del alumnado, y favorecen las relaciones entre los miembros del grupo. La comunicación debe entenderse como elemento global que determina esas relaciones. El cuerpo y sus recursos expresivos, en el proceso de elaboración e interpretación de mensajes, han de contemplarse en un contexto comunicativo de forma simultánea con otros códigos y lenguajes. Conviene explorar diferentes posibilidades y recursos expresivos del propio cuerpo, utilizándolos en danzas, mimos, representaciones, etc. apreciando la calidad del movimiento y los diferentes componentes del mismo. La adaptación de la expresión corporal a tiempos determinados supone ajustes corporales a diferentes frecuencias, ritmos variados, bailes, etc. conjuntado la música, el movimiento y la propia expresividad en relación con actitudes, sensaciones y estados de ánimo.

El enriquecimiento de las posibilidades de comunicación en las relaciones con los otros, desarrollará en el alumnado de Educación Secundaria Obligatoria el sentido de la responsabilidad dentro del grupo, mejorará su capacidad de integración social y potenciará el respeto a la libertad y a las normas establecidas.

\section{4.-LAACTIVIDAD FÍSICAENELMEDIONATURAL}

LaEducación Física debe incorporar contenidos que desarrollen la sensibilidad del alumnado con respecto al medio natural, fomenten su conocimiento y respeto y

favorezcan su disfrute. Se hace, pues, necesario conocer normas, medidas y reglamentos para la realización de actividades en la naturaleza. El estudio de las características del medio natural a través del aprendizaje de técnicas de orientación, topografía y de reconocimiento de formas y elementos del paisaje, harán posible su adaptación al medio.

$\mathrm{Al}$ tratarse de un entorno no habitual se requiere un conocimiento de las normas específicas de seguridad, protección y precauciones que se tendrán en cuenta en la utilización y disfrute del medio natural. Deberá informarse sobre lugares, zonas, instalaciones de permanencia, parques, reservas naturales, dónde se podrán realizar actividades de este tipo.

\subsection{Evolución y corrientes de epistemológicas de contenidos del currículum.}

Los contenidos de EF han evolucionado en su historia basados en diversos factores. Así, siguiendo a Rodríguez (2003), los factores de evolución han sido factores Externos (son consecuencia de intereses políticos, sociales, económicos, etc.) y factores Internos (que están vinculados a la institución escolary a las exigencias que esta ejerce sobre las materias que componen su currículum.

Hernández (citado en Contreras, 1998), establece en tres los factores responsables de la evolución de los contenidos:

1. La Construcción histórica de la asignatura de EF: En un momento determinado la idea de cuerpo-máquina dio lugar a un concepto de la $\mathrm{EF}$ de carácter instrumental, donde la condición física era el contenido hegemónico.

2. El papel del profesor: Este factor alcanza la introducción de aquellos contenidos que permiten desarrollar unos valores educativos acordes a su formación. Según Contreras (1998), es el caso de la psicomotricidad, que supuso un nuevo estatus para los profesionales de nuestra área, puesto que relaciona el movimiento con los aprendizajes escolares básicos.

3. Las expectativas de la sociedad: Los agentes sociales, alumnado, profesores, padres y madres... y sobre todo la influencia televisiva del deporte, han supuesto un muchos casos un freno a la incorporación de nuevos contenidos en nuestra área.

Una visión rápida y esquemática nos ofrece Blázquez (1995, p.85) sobre la evolución de los contenidos de la EF:

\begin{tabular}{|c|c|}
\hline OBJETIVOS & CONTENIDOS \\
\hline $\begin{array}{l}\text { 1. Higiene postural y respiratoria } \\
\text { - Disciplina fisica y moral. }\end{array}$ & - Gimnasia Sueca y Neosueca. \\
\hline \multicolumn{2}{|c|}{ INDEPENDENCIA CON EL DEPORTE } \\
\hline $\begin{array}{l}\text { 2. Mens sana in corpore sano } \\
\text { - Inculcar el juego limpio "fair play". }\end{array}$ & $\begin{array}{l}\text { - El deporte en la escuela como medio de } \\
\text { formación integral. }\end{array}$ \\
\hline \multicolumn{2}{|c|}{ VINCULACIÓN TOTAL AL DEPORTE } \\
\hline $\begin{array}{l}\text { 3. Rendimiento físico. } \\
\text { - Salud física. }\end{array}$ & $\begin{array}{l}\text { - Trabajo sistemático de la condición física } \\
\text { "Physical Fitness". }\end{array}$ \\
\hline \multicolumn{2}{|c|}{ VINCULACIÓN INSTRUMENTAL CON EL DEPORTE. } \\
\hline $\begin{array}{l}\text { 4. Reeducación motriz. } \\
\text { - Ajuste perceptivo-motor. } \\
\text { - Relación interpersonal. } \\
\text { - Relación grupal y social. }\end{array}$ & $\begin{array}{l}\text { - Psicocinética. } \\
\text { - Psicomotricidad. } \\
\text { - Psicomotricidad relacional. } \\
\text { - Sociomotricidad. }\end{array}$ \\
\hline \multicolumn{2}{|c|}{ ESCASA VINCULACIÓN O RECHAZO AL DEPORTE } \\
\hline 5. Salud integral & - Aeróbic, Jogging, etc. \\
\hline \multicolumn{2}{|c|}{ VINCULACIÓN MARGINAL CON EL DEPORTE } \\
\hline
\end{tabular}

Pero vamos a detenernos más y para ellos vamos a seguir a Rodríguez (2003), quien entiende que la evolución de los contenidos de la EF pasa por:

\section{A) Primera Mitad del siglo XX}

Tomando como punto de inicio a principios del siglo XX, aparecen los movimientos gimnásticos, donde encontramos una evolución diferenciada de los contenidos dentro de cada movimiento en particulary de todos en general.

1. El movimiento del Centro:

- El movimiento rítmico de Dalcroze

- El nacimiento de la Gimnasia Moderna (Bode)

- La gimnasia natural escolar austriaca

\section{El movimiento del Norte:}

- Elli Börjsten, Niels Bukh y J. Lindhar, creadores de la gimnasia neosueca.

\section{El movimiento del Oeste:}

- Hebert crea el método natural basándose en las actividades del hombre primitivo en contacto con la naturaleza.

\section{B) Segunda Mitad del Siglo XX}

1. La etapa Higiénico-militar desde la guerra a 1965-70, tanto en Europa como en España se sufrió una guerra de forma consecutiva, la EF tiene un carácter militarista y de valores patrióticos. En el mejor de los casos se busca un componente higiénico para prevenir desequilibrios orgánicos, utilizando para ello la Gimnasia Educativa, basada en una sistematización de los ejercicios, de tipo anatómico-funcional. El trabajo viene estructurado siguiendo una organización racial y analítica de los ejercicios, basada en la gimnasia sueca, cuyo principal objetivo es perfeccionar de una forma equilibrada la fuerza, la flexibilidad, el desarrollo corporal, de forma metódica, racial y disciplinada. 
Agosti (1963) clasifica los ejercicios según su finalidad, y podemos observar la clase de objetivos y la selección de contenidos

- Grupo 1: Ejercicios de orden

- Grupo 2: Ejercicios elementales

- Grupo 3: Ejercicios fundamentales de piernas

- Grupo 4: Ejercicios de equilibrio

- Grupo 5: Ejercicios fundamentales de brazo

- Grupo 6: Ejercicios fundamentales de tronco

- Grupo 7: Ejercicios de locomoción

- Grupo 8: Ejercicios de salto

- Grupo 9: Ejercicios funcionales

-Grupo 10: Ejercicios discrecionales

El elemento de organización básica es la sesión y ésta se estructura en tablas de ejercicios ordenados de la siguiente manera:

- Parte Inicial: ejercicios dinámicos de activación (marcha, carrera, ejercicios de orden)

- Partes Fundamentales: trabajo localizado (analítico de las diferentes regiones corporales) y generalizado (sintético, de locomoción, saltos, aplicación, agilidad, deportes, danzas, juegos, etc.).

- Partes Finales o calmantes: marcha calmante y ejercicios de brazos.

\section{C) La Etapa Pedagógica de 1970-1980}

En esta etapa es cuando se va a producir un cambio en la concepción de la EF en España, en parte debido a las aportaciones provenientes del exterior, de teóricos como Le Boulch, Lapierre, Aucouturier, Picq y Vayer, etc., y de la aportación del fenómeno deportivo, liderado por la difusión de los JJOO, a través de los medios de comunicación, en especial la TV. A esto se le añade la introducción de las diferentes corrientes relacionadas con la expresión corporal y las aportaciones profesionales de la EF que surgen de los diferentes centros de formación del profesorado de EF, que al incorporar estos contenidos en su currículum, difundirán los mismos en los diferentes centros educativos en los cuales desarrollarán su labor.

Este cambio de planteamiento, pretende la formación completa del individuo atendiendo al campo motor, afectivo y social, por medio de la introducción de contenidos relacionados como:

- Los Deportes (fundamentalmente en su vertiente agonística, donde prima la correcta ejecución técnica y la elaboración de progresiones didáctica para su adquisición) es la corriente deportiva: deportes individuales, de adversario, de oposición, de combate, de cooperación y de cooperación-oposición.

- La Psicomotricidad, la EF de Base (basada en la concepción psicosomática de la persona, que van a generar teorías como la psicocinética, la psicomotricidad relacional, etc., que pretenden la educación del niño de forma integral a través del movimiento, con respecto a su entorno, a sí mismo y a los otros, en busca de una verdadera autonomía personal). Es la corriente psicomotricista y según Le Boulch se trabajarán: ejercicios de coordinación motriz, de estructuración del esquema corporal, de ajuste postural, de percepción temporal, de percepción del espacio y estructuración espacio-temporal y de actividades libre y juego.

- La Expresión Corporal (propone el concepto de «vivencia corporal», donde prima el desarrollo de la creatividad, el cuerpo vivenciado), llegando a las corrientes Expresivas. Según las corrientes expresivas, tenemos diferentes propuestas de contenidos:

*Corriente pedagógica: ejercicios estructurados para facilitar el aprendizaje.

*Corriente escénica: basada en la representación y en las diferentes técnicas escénicas usadas en el teatro, mimo, danza, etc.

*Corriente psicoanalítica: basada en las posibilidades terapéuti-

cas de las técnicas de grupo, catarsis individuales y colectivas, etc.

*Corriente metafísica: donde primar lo espiritual y lo místicoreligioso.

\section{D) La etapa de la década de los 80-90}

Se produce por una parte, una generalización del profesorado de EF con estudios superiores, ya sea directamente como licenciados o convalidados por medio de los procedimientos habilitados por la admi- nistración. Por otra parte, el hecho de la convocatoria de las primeras oposiciones de Profesores de EF en las EEMM, supone una evolución basada en la práctica y en la propia reflexión, dando un posicionamiento mayor y más estable al docente, afianzando los contenidos de EF curriculares en las etapas medias. A todo lo anterior, debemos añadir la importancia que como fenómeno social, alcanzan las actividades físicas en la ocupación del tiempo libre y de ocio, a nivel social apareen modas como el jogging, trekking, etc. Esta importancia social deriva en la implicación de la escuela en la educación del tiempo libre y de ocio.

De todo lo anterior surgen nuevos contenidos que se van incluyendo en elárea, contenidos que deben de desarrollar diferentes objetivos de los diferentes niveles de concreción, entre los que encontramos:

- Las Habilidades Motrices Básicas: aparecen como una alternativa menos ambiciosa, más pragmática y funcional que la psicomotricidad, en la cuál se apoya para dotar al individuo de un acervo motor importante.

- El Deporte: sus antecedentes son los juegos y las posibilidades de ocio de las sociedades. Aunque cuando hablamos del deportes moderno, nos referimos a una institución aparecida en Inglaterra durante el siglo XVIII llamada deporte, que integra las ideas de igualdad de oportunidades, fair play, reglamentos, ligas, árbitros, etc. Con su evolución, pasa de ser agonista a contemplarse desde tres puntos de vista diferentes:

*Deporte Institucional: es el deporte competitivo, institucionalizado (federado), donde se busca el máximo nivel de ejecución y obtener deportistas del mayor nivel posible.

*Deporte Recreativo: el deporte aparece como medio de diversión y de entretenimiento, el hecho de ganar o perder pierde su función, y donde lo importante es la diversión y las necesidades de movimiento, se adaptan las reglas a las necesidades del momento. *Deporte Educativo: surge del rechazo de los modelos mecanicistas imperantes en la enseñanza de los deportes. Se modifican en base a criterios educativos, estructurando los deportes y sus estructuras en función de las necesidades educativas del alumno.

- La Expresión Corporal: el origen de esta corriente en su manifestación como medio de comunicación se remonta a las culturas primitivas, evolucionando hasta consolidarse con la corriente rítmica de Delsarte (1811-1871), Duncan (1878), Bode (1881-1968) y Jalkanen (1889-1964). En el actual modelo curricular se implanta como bloque de contenidos propio en su modalidad pedagógica, cuyo objetivo es posibilitar la seguridad, el autoconocimiento expresivo, la desinhibición de los alumnos, etc. Las manifestaciones más innovadoras en EF las encontramos en la gimnasia-jazz, el aeróbic, la danza tradicional y popular, el baile en todas sus manifestaciones, etc.

- Los Juegos: pasan a tener un estatus principal donde su carácter flexible, lúdico y socializante lo hacen necesario enEF Amediados de la década de los 80 , se procede a la experimentación de la Reforma de las EEMM, donde se establecen centros que con carácter experimental y de forma voluntaria. Aceptarán adelantar los postulados de la reforma, se establecen calendarios de reuniones de los diferentes equipos de profesores de carácter interdisciplinar y de área, discutiendo y definiendo diferentes líneas de actuación sobre la base de los posturales de la reforma.

- Las Actividades en la Naturaleza: especial importancia tuvo el movimiento Scout iniciado en 1907, entendiendo estas actividades como medio formativo para los jóvenes. En la actualidad, las Actividades en la Naturaleza constituyen un conjunto de prácticas educativas y recreativas, que surgen en los países desarrollados en la década de los setenta, se promocionan y extienden en los ochenta y se consolidan en la última década gracias a los nuevos hábitos y gustos de la sociedad postindustrial. Su gran valor educativo queda demostrado por trabajos de Rousseau y otros, recogidos en Baena (2005):

- Socialización, valores medioambientales, emancipación, superación, igualdad entre sexos, fantasía, etc. (Fernández-Quevedo y cols.; 2001)

* Valores lúdicos y de entretenimiento: Rodríguez García, 1995. 
*Valores Psicoemocionales: Buscar sensaciones (bienestar, placer, nerviosismo, autosuperación, etc. (Zuckerman, 1979, Heyman y Rose, Martos, 1999; etc.).

*Valores Higiénico-saludables: Buscar efectos positivos para la salud. El bienestar persona y grupal. (Baena, 2006).

\section{E) De la Reforma hasta nuestros días}

La implantación de la Reforma Educativa con la promulgación de la LOGSE, estructura los contenidos de la ESO, en 5 bloques y en 4 los de Bachillerato. Se fijan así unos «mínimos» (objetivos, contenidos, criterios de evaluación, etc.) a impartir en todas las comunidades autónomas para garantizar una formación mínima común en todo el territorio español. Este sería el Primer Nivel de Concreción, propuesto por la Administración Educativa Central que aporta los decretos de por los que se establecen las enseñanzas mínimas:

- Real Decreto 1007/1991 de 14 de Junio para la ESO, modificado por el Real Decreto 3473/00.

- Real Decreto 1178/1992 de 2 de Octubre para Bachillerato, modificado por el Real Decreto 3474/00.

De los cuales parte la CCAAAndaluza para publicar sus Diseños Curriculares Bases de cada etapa a través del D.106/1992 modificado por el 148/2002 para la ESO, por el que se establecen las enseñanzas correspondientes a la Educación Secundaria Obligatoria en Andalucía. Dentro de los cuales se distinguen: objetivos, contenidos, metodología y evaluación. En relación a la LOCE (Ley de Calidad 10/2002 de 23 de Diciembre) se aprobó el Real Decreto 831/03 para ESO y el Real Decreto 832/2003 para Bachillerato.

Actualmente en la ESO el área de EF se encuentra regulada por varias normas. En los cursos primero y tercero de la ESO, y siguiendo la Orden 10 de Agosto de 2007 por la que se desarrolla el Currículo correspondiente a la ESO en Andalucía, nuestro referente currícular de momento sería el RD 1631/2006 de 29 de Diciembre por el que se establecen las enseñanzas mínimas correspondientes a la ESO.

3.2. Valor educativo de las corrientes epistemológicas del área de Educación Física.

Consideramos la década de los sesenta como el punto de referencia para analizar la aparición de las nuevas tendencias en Educación Física que llegan hasta nuestros días. Lógicamente, nacen como resultado de nuevos estudios e investigaciones basadas en las corrientes de principios de este siglo que son analizadas por numerosos autores (Loza, 1993; Del Moral, 1993; Cecchini, 1996, etc). La interpretación de las actividades motrices que han realizado nuestros antepasados, puede servimos para comprender y analizar nuestro momento actual.

Basándonos en Garrote (1993) consideramos que las tendencias actuales más significativas son: la deportiva, la psicomotora, la expresiva, la educación física de base, la investigadora en EE.UU. y la sociomotora. Se podrían, ampliar a otras cuatro que, por su escasa difusión en unos casos, o por su menor relación con el ámbito educativo en otros, creemos que son menos relevantes. Estas corrientes son: la corriente integradora de Gangey, el diseño curricular en Educación Física de G. Fernández y V. Navarro, el aeróbic y el Physical Fitness. Vamos a ver, las más importantes a nivel curricular:

\section{El Deporte Educativo}

El deporte está influenciado por la sociedad de consumo, es selectivo para la competición, y está comercializado. Desde esta perspectiva, no debe ser introducido en la escuela. Así, el deporte es criticado por sus métodos (analíticos, directivos, basados en la demostración y repetición) y por sus objetivos de competición y rendimiento. Por ello, autores como Devís y Peiro (1992) critican el deporte, considerándolo inadecuado como contenido curricular de la Educación Física.

Por otra parte, la presión social de los aficionados deportivos, de los políticos, de los medios de comunicación, y de los propios alumnos/ as, empujan a la inclusión del deporte en el curriculum.

Ante esta contradicción, se abre paso como vía de compatibilidad, la idea de «deporte educativo» defendida por numerosos autores de reconocido prestigio nacional e internacional como Sánchez Bañuelos (1986), Pierón (1988), Parlebás (1989) y Romero (1993). El deporte puede ser utilizado para conseguir fines educativos, utilizando métodos adecuados, acordes con las orientaciones de la actual reforma, estableciendo objetivos socializadores o planteando actividades motivantes y participativas. En definitiva, despojando al deporte de competición aquellos elementos que no le hacen adecuado para utilizarlos en el ámbito escolar. Resulta curioso descubrir que en 1949, P. Seurin, citado por Torres (2005), publica su obra «Hacia una educación física moderna», en la que intenta aunar la tendencia del Deporte de competición con laEducación Física antideportiva

\section{La Condición Física}

Esta tendencia de preocupación hacia la condición física está cobrando una importancia sociológica dentro $y$ fuera del ámbito educativo (Delgado, 1994). El estado de Condición Física-Salud y Fitness viene asociado a la salud y pretende la mejora del estado general de la persona física y psíquicamente. Trata de aprovechar al máximo el potencial del organismo, no sólo a través del ejercicio físico sino también de la dieta, el estilo de vida, la actividad mental, et sueño, etc... Por tanto, va más allá del ejercicio, tratando de adquirir una actitud más positiva hacia la vida. Dentro de los contenidos a trabajar, podemos establecer un gran elenco que van desde el trabajo biológico, hasta el cuidado del cuerpo.

\section{La Psicomotricidad}

Su importancia en el campo de la Educación en general, y de la Educación Física en particular, es enorme, pues descubre todo un mundo de acción reeducativa y educativa a través del movimiento, aportando unos contenidos sin los cuales no se entendería laEducación Física en la actualidad.

Autores como Wallon, Ajuriaguerra o Guilmain desarrollaron numerosas investigaciones y han publicado valiosas aportaciones en esta línea. El apoyo desde la psicología evolutiva de Piaget, Gessel, Spitz o Lezine ha facilitado laaceptación del papel fundamental de lamotricidad en el desarrollo integral de la persona.

La inclusión definitiva de la psicomotricidad en el campo educativo no se produce hasta la década de los 60, en que aparece el trabajo de Picq y Vayer (1969) y a partir de ese momento comienzan determinadas tendencias que Garrote divide en tres:

\section{a) Aproximación psicopedagógica de Picq y Vayer.}

Estos autores critican las formas reeducativas de la gimnasia correctiva y la gimnasia rítmica, por considerarías contrarias a la esencia motriz. La educación psicomotriz es una «acción pedagógica y psicológica que utiliza los medios de la Educación Física con el fin de normalizar o mejorar el comportamiento del niño». La psicomotricidad para estos autores no es solamente un método, sino que alcanza la categoría de acción educativa global. Su principal aportación, desde el punto de vista psicopedagógico, consiste en haber fijado unos niveles de desarrollo psicomotor, debiéndose ajustar las tareas de aprendizaje a dichos niveles. Para Vayer (1972) la finalidad de la educación es la de guiar y favorecer el conocimiento personal a través de las relaciones que se establecen entre el «yo». Los«otros» y los «objetos». Este mismo autor comenta que para poder adaptarse a las diversas situaciones de ese mundo exterior y a sus eventuales modificaciones, el niño debe poseer la conciencia, el conocimiento, el control y la organización dinámica de su propio cuerpo. Ahora bien, la conciencia de sí mismo se adquiere de forma paralela a la conciencia del mundo circundante y a la vez la relación con el otro, que es el elemento fundamental del propio desarrollo de la personalidad.

b) Aproximación psicocinética de Le Boulch.

Su concepción del cuerpo humano (1978, p.274) surgida de los nuevos datos neurológicos, psicológicos y sociológicos le lleva a plantear una «ciencia del movimiento que parte de la existencia corporal como totalidad y como unidad» diferenciándola del ejercicio físico (gimnasia o deporte) mecánico y analítico, ya que éstos crean estereotipos y rigideces que anulan la capacidad de decisión de la persona.

Este profesor de Educación Física, médico y psicólogo entiende que, en la medida que el aprendizaje se aleja de la mecanización y se 
apoya en la vivencia, constituye un elemento enriquecedor del esquema corporal. El aprendizaje consiste, por tanto, en adquirir nuevos modos de acción que permitan al sujeto adaptarse a las diversas situaciones del medio y a sus propias disponibilidades.

Adiferencia de Picqy Vayer, el método psicocinétíco no parte de la reeducación, sino que supone un nuevo modelo de Educación Física Este método (1969) persigue un doble objetivo, por una parte el desarrollo de las capacidades motrices básicas y por otra, sentar las bases de otros aspectos de la educación como son los aprendizajes escolares. Este segundo aspecto ha dado la oportunidad a los profesionales de la Educación Física para justificar la igualdad de condiciones con otras asignaturas de tipo instrumental Esta postura es criticada por Arnaud (1983, citado por Garrote, 1993), porque desvirtúa los objetivos de la Educación Física.

c) La educación vivenciada de Lapierre y Aucouturier.

Esta tercera corriente, representada por Lapierre y Aucouturier (1977), utiliza el movimiento como medio de aprendizaje, a la vista del gran número de fracasos escolares en la educación infantil. Según Garrote (1993, p.32), estos autores parten de la unidad de la persona y consideran la acción motriz como medio de ir desarrollando todos los aspectos educativos del niño, tanto en el plano afectivo, como social e intelectual.

El niño es puesto en situaciones problema, en las que debe descubrir por sí mismo, orientado por el profesor/a.

Al igual que Vayer, estos autores consideran el concepto de «educación vivida» como lo esencial de la educación de los niños pequeños. De esta forma, surge la psicomotricidad como un fenómeno educativo dentro del contexto general de la enseñanza, sin tener como objetivo la adquisición de gestos automáticos, de estilos y de técnicas deportivas. En la educación psicomotora, el movimiento no es más que un soporte que permite al niño adquirir conceptos abstractos, percepciones y sensaciones que le brindan el conocimiento de su cuerpoy, a través de él, el conocimiento del mundo que le rodea.

Sin embargo, González (1993), apoyándose en otros autores como Cratty, Singer o Sánchez Bañuelos, critica las tendencias psicomotrices o psicocinéticas por considerar que aunque propician una serie de experiencias y vivencias, en definitiva, no enseñan nada. Denuncia la pobreza de ejecución motriz espontánea que existe en la población infantil.

\section{La Expresión Corporal}

Utilizar el movimiento como medio de comunicación es una de las corrientes actuales de la educación física. Históricamente la danza $y$ la comunicación no verbal se remontan a culturas primitivas y han ido evolucionando hasta consolidarse en la corriente rítmica, destacando nombres como F. Delsarte (1811-1871), I. Duncan (1878-1929), Dalcroze(1865-1950) y, posteriormente, R. Bode (1981-1968) o H Jalkanen (1889-1964).

En los años sesenta, la oposición frontal al deporte y la nueva concepción del movimiento como medio educativo, iniciado por Le Boulch, significa un auge de las distintas formas de Expresión Corporal

Dentro del curriculum de la Educación Física ha quedado implantado un nuevo contenido que recibe el nombre de «expresión corporal».

Pueden existir distintas modalidades de Expresión Corporal como la escénica, el psicoanalítico, la metafísica y la pedagógica. Estaúltima es la que más nos interesa en Educación Física. Se busca que el alumno/a descargue sus energías a través del juego corporal, que encuentre los medios auténticos de expresión creadora correspondientes a su edad, estimulándole el deseo de descubrir, conocer y utilizar cada vez mejor sus aptitudes y aplicarlas en su vida diaria, como señalan Schinca (1988) y Stokoe (1988). Torres (2005) habla de dos vertientes: el movimiento rítmico codificado (danza en sus múltiples acepciones) y el movimiento expresivo puro.

\section{La Educación Física de Base.}

LaEducación Física de Base es una forma de acercarse al movimiento utilizándolo como instrumento educativo, con el objetivo de mejorar la capacidad de percibir, de tomar decisiones, y de ejecutar los movimientos desde los más sencillos a los más complejos. Adiferencia de Le
Boulch, Legído considera que este medio es aplicable, no sólo en el ámbito educativo, sino también como base del perfeccionamiento deportivo. Las técnicas deportivas son adquiridas a partir de los patrones motores básicos que pueden ser enriquecidos con múltiples y variadas experiencias motrices, y no a partir de repeticiones mecanicistas o de patrones fijos de movimiento.

\section{La Corriente Didáctica}

Guiados por la psicología conductista, un grupo de expertos coordinados por Bloom fijó en tres los ámbitos educativos: cognitivo, afectivo y motor. Fue Harrow quien, en 1978 publica su «Taxonomía del ámbito psicomoton» considerada como la más completa. En la vertiente metodológica, el autor más representativo es Mosston con sus dos libros anteriormente mencionados.

En España, nuestro máximo representante de esta corriente ha sido Delgado, seguido por otros como Devís y Devís, Contreras, Pierón entre otros. La preocupación y por tanto el tratamiento educativo más importante de esta corriente se ha centrado en la didáctica de la enseñanza. Todo se ubica alrededor de la importancia de los Métodos de Enseñanza, los Estilos de Enseñanza, las Estrategias en la Práctica, los Recursos Didácticos, etc., que serán las que llevarán al éxito educativo. Se centra principalmente en el docente y en su forma de actuar en el acto de enseñanza, valorando e investigando la eficacia de su acción.

\section{La Corriente Sociomotriz}

El término «movimiento» lo sustituye Parlebás por «conducta motriz», por considerarlo más adecuado al llevar implícito el hecho educativo e integral de la persona. La noción de movimiento reduce la acción física a las características de desplazamiento de la máquina biológica e hipervaloriza de modo abusivo la descripción técnica.

La construcción de una nueva ciencia para la Educación Física sería para Parlebás la solución a la actual confusión que existe en los métodos, técnicas y doctrinas. Este autor opta por un análisis de la motricidad humana como «inteligencia motora», la cual aporte nuevos esquemas de movimiento en situaciones desconocidas creando una estructura intelectual cada vez más compleja, como por ejemplo las situaciones problema de los juegos colectivos.

Considera Parlebás (1989) que la Educación Física no es la prolongación de otras disciplinas, sino que posee su propia identidad. Sin embargo, Vázquez (1989) critica esta aseveración por considerar que es contradictorio pretender una ciencia de la Educación Física y nutrirse de elementos teóricos de otras disciplinas para su fundamentación.

\section{Conclusiones.}

La epistemología del área de Educación Física, es una fuente imprescindible y evidente del proceso curricular, el cual fluctúa y varía a lo largo del tiempo. Las necesidades específicas de cada área, deben tener una estrecha relación con la fundamentación del conocimiento científico y el entorno social al que sirve, puesto que la selección, secuencia y organización de contenidos, debe ser una respuesta a la necesidad social de los alumnos.

La aparición de continuas y no mejoradas leyes educativas, están produciendo una herida en la estructura epistemológica del área cada vez más latente, dejando muchos contenidos sin incluir en las nuevas currículas y retrasando en aprendizaje en los años de los alumnos de otros que se consideran imprescindibles y que no van en armonía con las referencias sociales del siglo XXI.

\section{Referencias Bibliográficas.}

Agosti, L. (1963). Gimnasia educativa. Madrid: Luis Agosti.

Aucoutier, B. \& Lapierre, A. (1977). La Educación Psicomotriz como terapia. Barcelona: Médica y Técnica.

Baena, A. (2005). Bases teóricas y didácticas de la Educación Física escolar. Granada: Gioconda.

Arnold, P.J. (1991). Educación Física, movimiento y currículum. Madrid: Morata 
Baena,A. (2006). Actividades en el medio natural. Tipos, clasificaciones y recursos. Organización de actividades fisica en la naturaleza. Granada: Inédito.

Blázquez, D. (dir.)(1995). La iniciación deportivay el deporte escolar. Barcelona: Inde.

Bourdieu, P.; Chamboredon, J.C.; Passeron, J.C. (1976). Sociología del conocimiento y metodología. Argentina: Siglo XXI.

Bunge, M. (1985). La investigación cientifica. Barcelona: Ariel.

Cagigal, J.M. (1968). La Educación Física, ¿ciencia?. Rev. Citius, Altius, Fortius, $\mathrm{n}^{\circ} \mathrm{X} 1,5-26$.

Cecchini, J.A. (1996). Epistemología de la Educación Física. En V. García Hoz, Personalización en la Educación Física. Madrid: Rialp.

Coll, C. (1992). Psicología y currículum. Barcelona Laia.

Contreras, O. (1998). Didáctica de la Educación Física: un enfoque constructivista. Barcelona: Inde.

Delgado, M. (1994). La saluden la Educación Primaria. Sevilla: Dpto. Expresión Musical, Plástica y Corporal. Sevilla.

Decreto 148/2002, de 14 de mayo, por el que se modifica el Decreto 106/1992, de 9 de junio, por el que se establecen las enseñanzas correspondientes a laEducación Secundaria Obligatoria en Andalucía.

Decreto 231/2007 por el que se establece la ordenación y las enseñanzas correspondientes a la Educación Secundaria Obligatoria.

Devís, J. \& Peiro, C. (coord.) (1992). Nuevas perspectivas curriculares en Educación Física: la saludy los juegos modificados. Barcelona: Inde.

Diaz Lucea, J.(1994). El currículum de la EF en la Reforma Educativa. Zaragoza: Inde.

Diccionario de Ciencias de la Actividad Física y el Deporte (1998). Barcelona: Paidotribo.

Fernández-Quevedo, C.; Pasamontes, M.J. \& Del Campo, J. (2001). Las Actividades en la naturaleza en Primaria. Lecturas Educación Física y Deporte, Revista Digital [en línea], 7, 38. Buenos Aires. Disponible en: http://www.efdeportes.com [Consulta: 2005, 23 de Febrero].

Fernández, G. \& Navarro, V. (1989). Diseño Curricular en Educación Física. Barcelona: Inde.

Garay, J. \& Hernández, A. (2005). La actividad física y el deporte en el marco científico». Lecturas Educación Física y Deporte, Revista Digital [en línea], 10, 85.Buenos Aires. Disponible en: http:// www.efdeportes.com [Consulta: 2006, 16 de Marzo].

Garrote, N. (1993). Educción Física y su contexto, En V. Martínez, La Educación Física en Primaria. Reforma de 6 a 12 años. Barcelona: Paidotribo.

Gimeno,A.(1998). Cap. I: Fundamentación epistemológicay curricular. Educación Física en Primaria. Fundamentación y desarrollo curricular. Barcelona: Paidotribo.

González, F. (1993). Temas transversales y educación en valores. Madrid: Alauda. Madrid.

Gutiérrez, M. \& Oña,A. (2003). Apuntes de la Asignatura de Métodos ytécnicas en la Ciencia de la Actividad Físicay del deporte. Inédito: Granada.

Heyman, S. R. \& Rose, K. G. (1979). Psychological variables affecting scuba performance. En, C. H. Nadeau, W. R. Halliwell, K. L. Newell \& G. C. Roberts (Eds.), Psychology of Motor Behavior and Sports. Champaign, IL: Human Kinetics Press.

Krech, D.; Crutchfield, R.; Ballachey, E. y Álvarez Villar, A. (1978). Psicología social. Madrid: Biblioteca nueva.

Lagardera, F. (1989) Educación Física sistemática: hacia una enseñanza contextualizada. Apunts, Educación Física y Deportes, 16, 29-36.

Le Boulch, J. (1978). La educación por el movimiento en la edad escolar. Buenos Aires: Paidós.

Ley Orgánica 2/2006, de 3 de Mayo de Educación.

Ley Orgánica 10/2002, de 23 de diciembre, de Calidad de la Educación.

Ley Orgánica 1/1990 de 3 de Octubre de Ordenación General del SistemaEducativo.
Martos, C. (1999). Contributos para a compreensao dos desportos radicls. Revista de Ciencias do Desporto. Ludens, 2, 53-63.

Orden 10 de Agosto del 2007 por la que se desarrolla el currículo correspondiente a la Educación Secundaria Obligatoria en Andalucía

Osgood, C.E. (1949). The similarity paradox in human learning: a resolution. Psychological Review, 56, 132-143.

Parlebás, P. (1981). Contribución al léxico en las ciencias de la acción motriz. París: INSEP.

Parlebás, P. (1989). Perspectivas para una Educación Física moderna. Cuadernos Técnicos $n^{\circ} 1$. Málaga: Unisport.

Pedráz, V. (1988). Teoría Pedagógica de la Actividad Física. Madrid: Gymnos.

Pierón, M. (1998). Didáctica de las actividades fisicas y deportivas. Madrid: Gymnos.

Picq, L. y Vayer, P. (1969). Educación Psicomotriz y retrason mental. Barcelona: Científico Médica.

Real Decreto 3473/2000, de 29 de diciembre, por el que se modifica el Real Decreto 1007/1991, de 14 de junio, por el que se establecen las enseñanzas mínimas correspondientes a la educación secundaria obligatoria.

Real Decreto 3474/2000, de 29 de diciembre, por el que se modifican el Real Decreto 1700/1991, de 29 de noviembre, por el que se establece la estructura del bachillerato, y el Real Decreto 1178/1992, de 2 de octubre, por el que se establecen las enseñanzas mínimas del bachillerato.

Real Decreto 831/2003 de 27 de Junio por el que se establece la ordenación general y las enseñanzas comunes de la ESO (BOE 158 de 3 Julio).

Real Decreto 832/2003 de 27 de Junio por el que se establece la ordenación general y las enseñanzas comunes a Bachillerato (BOE 159 de 4 Julio).

Real Decreto 1631/2006 de 29 de Diciembre, establece las enseñanzas mínimas de Educación Secundaria.

Rodríguez, P. (1995). Deporte y Ciencia. Barcelona: Inde.

Rodríguez, J. (2003). Epistemología de la Educación Física. Inédito. Granada.

Sánchez Bañuelos, F. (1986). Bases para una Didáctica de la Educación Física y el Deporte. Madrid: Gymnos.

Sáenz-López, P. (1997). La Educación Física y su Didáctica. Cádiz: Wanceulen.

Sarabia, B. (1992). Los contenidos de la Enseñanza. Madrid: Santillana.

Stokoe, P. (1988). La expresión corporal. Arte, salud y educación. Buenos Aires: Humanitas.

Torres, J. (2005). Curso de Formación de Funcionarios de Educación Física en Secundaria. Granada: CEP.

Vázquez, B. (1989). La Educación Física en la Educación Básica. Madrid: Gymnos.

Vayer, P. (1972). El equilibrio corporal. Madrid: Científico-Médica.

Zuckerman, M. (1988). Behavior and Biology: Research on Sensation Seeking and Reactions to the Media. En, Communication, Social Cognition and Affect (pp. 173 - 194 ). Hillsdale NJ: Erlbaum.

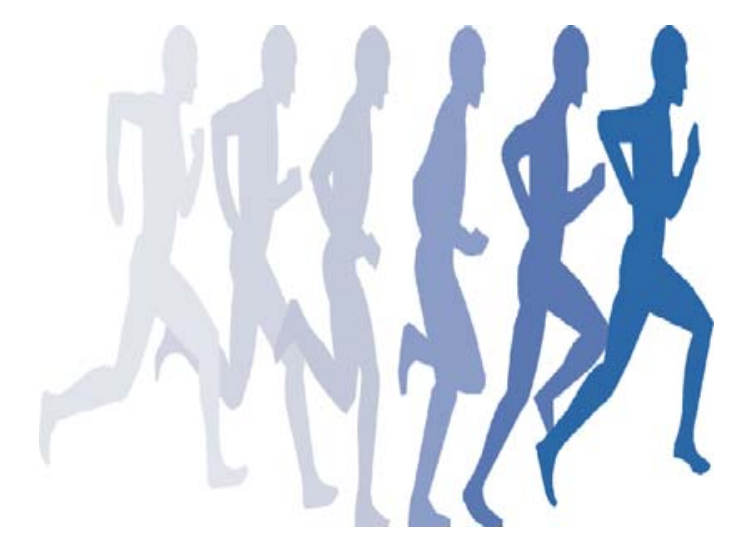

